\title{
Quotas for CFE Treaty \\ Declared Site Inspections for \\ Baseline Validation
}

DE91 002576

\author{
R. Scott Strait and Alan Sicherman \\ Treaty Verification Program \\ Lawrence Livermore National Laboratory
}

\section{Executive Summary}

The CFE Treaty will provide for limits on NATO and WTO forces, particularly tanks, armored personnel carriers, artillery, and helicopters. In addition to the overall limits on TLEs in the ATTU zone, there are expected to be secondary limits on single country forces, limits on forces based in foreign nations, and geographic sublimits. To help validate WTO declarations of baseline forces, the treaty may provide for on-site inspections by NATO of declared WTO basing facilities. One important unresolved issue concerning haseline declared-site OSIs is the quota of such inspections allowed each country. This report presen 15 a decision analysis and evaluation in support of recommendations for resolving this and related issues. It also identifies key policy decisions that impact the determination of the number of declared-site OSIs. These decisions are:

- Desired probabilities of detecting a violation and of falsely accusing WTO;

- Trade-off between improved verification and the intrusiveness of additional OSIs;

- Force strength constituting a militarily significant violation; and

- Degree of coordination with and reliance on inspections by NATO allies.

In addition to declared-site OSIs, inspections of non-declared sites, aerial inspections, and NTM will also be useful in the baseline validations. The U.S. and NATO abilities to monitor WTO compliance depends on joint capabilities of all monitoring sources. No single verification measure should be relied upon to monitor WTO compliance. They work together in a direct way to improve NATO and U.S. estimates of force strength. Perhaps more importantly, each has different vulnerabilities to spoofing. These differences greatly increase the difficulty for WTO in evading detection of violations of baseline declarations. For example, declared site OSIs can be timed to coincide with NTM coverage so that attempts to conceal TLEs immediately before the inspection will be detected. Similarly, aerial inspections and OSIs can be appropriately timed. Also inspections of different declared sites, or declared sites and undeclared sites can be planned to alert NATO to WTO spoofing of inspections.

In addition to intentional spoofing by WTO, inspections are subject to uncertainties in their estimates due to the inexact nature of their counts. These uncertainties could lead to either overestimates or underestimates of forces. It is unlikely that an inspection team will be able to actually count each TLE at a site, because of time and budget constraints and because force movements may confuse the counting (even if that is not the WTO intention). Using sample counts, overviews and other indications of force strength, the forces at a site will be estimated.

In attempting to incorporate into our analysis as many important realities of the problem as possible, we have made several assumptions as a base case. Later in this report, we will explore the sensitivities of the results to variations in these assumptions. We have assımed 2500 WTO declared sites with TLEs and that 10\% excess of any type of TLE over the total WTO declaration is the minimum militarily significant violation. We have assumed that excess TLEs would be spread 
evenly throughout the WTO bases in proportion to the declarations. This assumption is conservative in the sense that it maximizes the Soviet ability to evade detection.

In our analysis we represent the uncertainty surrounding an inspection by a probability distribution on the estirnated number of forces given the actual force strength. In our base case, the uncertainty in the estimate from an OSI of WTO forces at a single base has a 0.25 ratio of the standard deviation to the mean. This implies, for example, that if the true number of tanks was 400 , then there is a $68 \%$ probability that the NTM estimate would be between 300 and 500 .

In order to evaluate the relative benefits and costs of declared-site OSIs, it is important to quantify the relative improvements in U.S. monitoring ability of additional inspections. These improvements can be quantified as reductions in the probabilities of missed violations and false accusations. The results of the base case analysis are presented in Fig. ES-1 below. Note that as few as 50 baseline OSIs can achieve a monitoring capability with less than $10 \%$ of either missing a militarily significant violation or signalling a false alarm. These results assume that the OSIs are coordinated with aerial inspections, NTM, and other monitoring measures as necessary to prevent WTO spoofing of the inspection. As illustrated, more inspections always provide improvements in monitoring capabilities, but with diminishing improvements in monitoring ability for each additional inspection. Reductions in the uncertainties surrounding OSIs, and correspondingly in the required number of OSIs, may be achieved by having each OSI accompanied by an aerial inspection.

\section{Figure ES-1.}

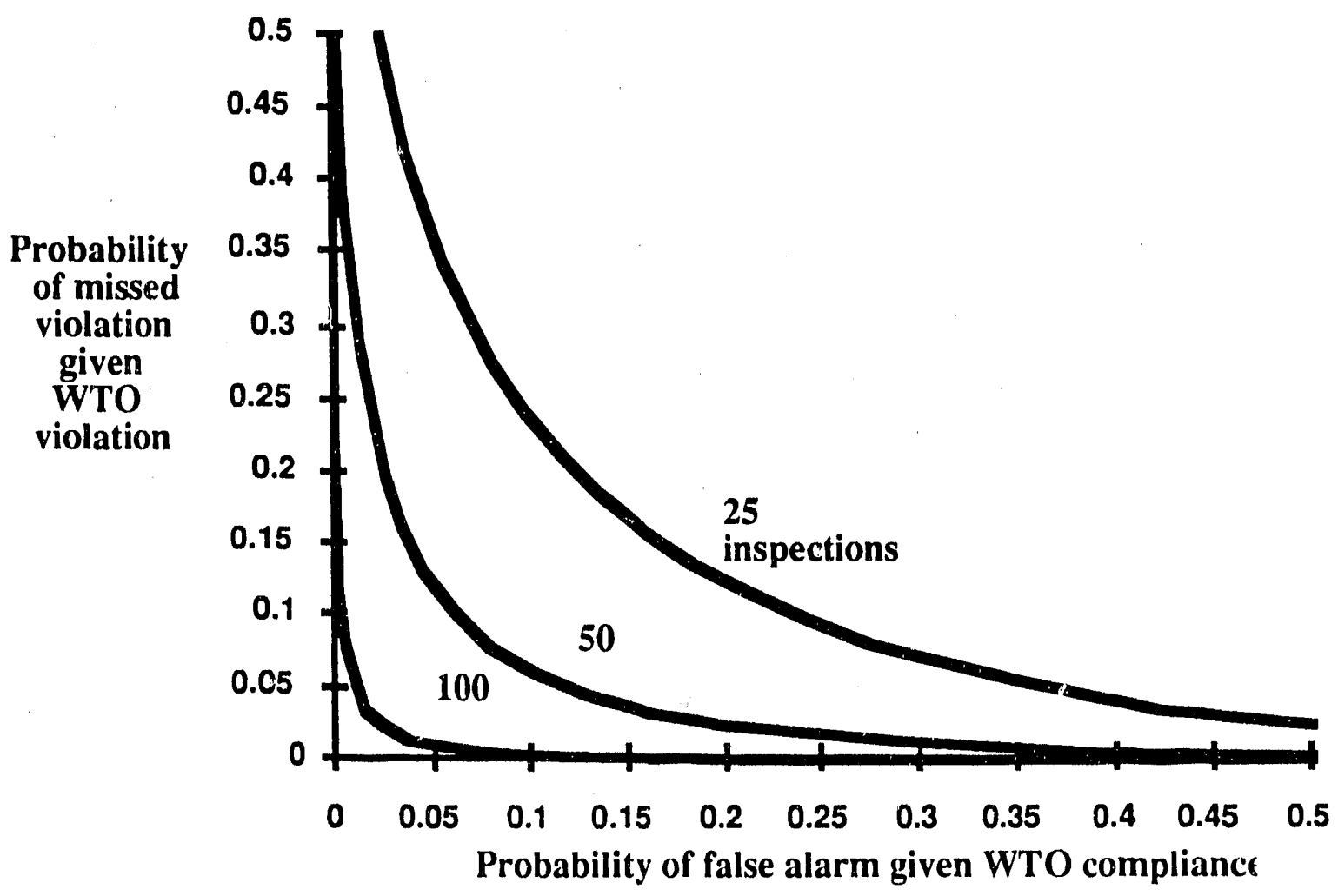


Also presented in the report are the results when assumptions about the following factors are varied: - Uncertainty in inspection estimates;

- WTO ability to spoof inspections; and

-WTO strategies for evading detection and allocating violations among bases.

Several recommendations are presented concerning the inspection procedures and a discussion is included about the methodology for actually selecting bases for inspection. A brief review of the application of this effort to annual continuing OSI quotas is also included.

The determination of the desired number of declared-site OSIs requires a mix of technical and policy judgments. This report presents an approach for incorporating the necessary technical judgments while leaving the policy judgments as parameters to be decided by policy-makers. The most important policy judgments are the choice of the required probabilities of detection and false accusations, and the trade-off of increased detection against the intrusiveness of additional inspections. A closely related input is the force strength constituting a militarily significant violation. Another critical judgment is the willingness of the U.S. to coordinate with and rely upon inspections carried out by NATO allies. Several other important inputs are highlighted in the report.

\section{Introduction}

The CFE Treaty will provide for limits on NATO and WTO forces, particularly tanks, armored personnel carriers, artillery, and helicopters. In addition to the overall limits on TLEs in the ATTU zone, there are expected to be secondary limits on single country forces, limits on forces based in foreign nations, and geographic sublimits. The treaty will provide for information exchanges, certain stabilization measures, and verification measures. The verification provisions should deter violations of the treaty, enable violations to be detected in timely fashion, and build confidence in the treaty. Throughout the life of the treaty, the verification measures focus on three related tasks. In chronological order they are: validate baseline data, monitor reductions, and confirm compliance with residual force limits.

The specific verification measures provided by the treaty to accomplish these tasks include declared site inspections, inspections of non-declared sites, and aerial inspections. In addition, the treaty will prohibit interference with NTM. The U.S. and NATO abilities to monitor WTO compliance depends on joint capabilities of all monitoring sources. No single veritication measure alone should be relied upon to monitor WTO compliance. The different verification measures complement each other, particularly, by covering each others' vulnerabilities.

OSIs at declared sites may be a major contributor to the validation of WTO baseline data, however, several important issues concerning these inspections remain unresolved. One of the most important unresolved issues is the quota of such inspections allowed each country. In choosing the required number of OSIs, the U.S. needs to balance the benefits of the number of inspections against their costs. The benefits of OSIs are generally the detection and deterrence of WTO violations and confidence building. The costs of the inspections are mostly their intrusiveness at NATO facilities and the financial expenditures. This report presents a decision analysis and preliminary evaluation of the benefit-cost tradeoffs for the baseline OSI quotas. In our analysis, we attempt to go beyond simple statistics and incorporate as many important aspects as possible that impact the validation of CFE baseline data. 


\section{Verification Environment}

There are three main aspects of the verification environment that affect the U.S. and NATO ability to validate WTO baseline data. One of these is the magnitude and type of the violation that is of concern to the U.S. A second aspect is the vulnerabilities and uncertainties of the different monitoring sources and, particularly, the OSIs themselves. In general, OSIs are subject to WTO spoofing, and furthermore, even in the absence of WTO spoofing. counts of TLEs will not be exact and completely accurate. The third aspect is the interactions and combinations of evidence from different monitoring sources. In this section, we will discuss each of these factors.

\section{Militarily Significant Violation}

It is important that the U.S. be able to detect and deter any militarily significant violation. The ability to detect a single excess tank out of the 40,000 to 50,000 currently possessed by WTO would be a nearly impossible task requiring highly intrusive verification provisions and significantly larger numbers of OSIs. While a single excess TLE may be politically significant, it would have negligible military significance. (This does not imply that the U.S. should not respond strongly to the detection of a small number of excess TLEs.) The choice of a militarily significant violation depends on a number of factors, including the relative importance of offensive versus defensive capabilities.

The violation of significance can be expressed in terms of the percentage of extra TLEs above the baseline declarations. For example, if the WTO baseline declaration were 40,000 tanks, then we might estimate that a militarily significant violation would be 4000 tanks or $10 \%$. Such a violation could be located at either declared or undeclared sites and allocated among the sites in a myriad of ways. From the WTO perspective, the smaller the violation, while still providing the desired military advantage, the lower the probability NATO will detect the violation, either with OSI, NTM, or other means. It is important to recognize that a certain percentage violation of the baseline declarations may translate into a rnuch larger percentage violation as forces are reduced according to the treaty. However, the larger the violation as a percentage of allowed forces, the less difficult its detection becomes.

In most cases, an important aspect of the military significance of a violation, is the time frame within which the U.S. receives warning of the violation. In validating the baseline data, the time frame is not a concern. The purpose of the baseline OSIs is to verify the declarations at a particular point in time, although as a practical matter a period of time, e.g., 60 days, will be allowed to accomplish this goal. However, the time frame may be critical in evaluating continuing annual quotas.

\section{Vulnerabilities and Uncertainties in the Monitoring Data}

The monitoring data from NTM, OSIs and other measures are all subject to both miscounts due to spoofing by the WTO and unavoidable inaccuracies in the NATO estimates of force strengths. In an effort to evade the treaty, WTO could purposefully cause NATO to undercount the number of TLEs. One approach to accomplishing this would be to conceal TLEs in excess of the baseline declarations. Such concealm.int could be done to spoof any of the verification measures available to NATO, although the range of measures available may make this difficult. If TLEs at a declared site were concealed at the time of an inspection the inspection force count may falsely validate the baseline declarations for that site. 
In addition to intentional spoofing by WTO, inspections are subject to uncertainties in their estimates due to the inexact nature of their counts. These uncertainties could lead to either overestimates or underestimates of forces. It is unlikely that an inspection team will be able to actually count each TLE at a site, because of time and budget constraints and because force movements may confuse the counting (even if that is not the WTO intention). Using sample counts, overviews and other indications of force strength, the forces at a site will be estimated.

In our analysis we will be represent the uncertainty surrounding an inspection by a probability distribution on the estimated number of forces given the actual force strength. As we explain in the next section, we can then compare the probability of an observed estimate given WTO compliance with the probability of the estimate given WTO violation. This comparison provides a measure of the strength of the evidence in favor of compliance or violation. WTO spoofing can be represented by basing the probability distribution of the evidence given violation on a lower force strength than the actual violation level. This approach allows us to evaluate a variety of different assumptions about the vulnerabilities and uncertainties surrounding OSIs.

\section{Interactions of Different Monitoring Sources}

The different monitoring sources all interact to create an overall verification framework. No single verification measure should be relied upon to monitor WTO compliance. They work together in a direct way to improve NATO and U.S. estimates of force strength. Perhaps more importantly, each has different vulnerabilities to spoofing. These differences greaily increase the difficulty for WTU in evading detection of violations of baseline declarations. For example, declared site OSIs can be timed to coincide with NTM coverage so that attempts to conceal TLEs immediately before the inspection will be detected. Similarly, aerial inspections and OSIs can be appropriately timed. Also inspections of different declared sites, or declared sites and undeclared sites can be planned to alert NATO to WTO spoofing of inspections.

Due to the complexity of these interactions, and their unique nature in each particular instance, analyzing these interactions is difficult. Our treatment of the uncertainty surrounding monitoring estimates, noted above and discussed more fully below, has the benefit of being able to combine the information from different sources and with different uncertainties according to the laws of probability. In the analysis presented below, we also point out how interactions among monitoring sources can affect the results in other ways.

\section{Methodological Overview}

In order to evaluate the relative benefits and costs of declared-site OSIs, it is important to quantify the relative improvements in U.S. monitoring ability of additional inspections. These improvements can be quantified as reductions in the probabilities of missed violations and false accusations. Both of these aspects of the problem need to be evaluated simultaneously because of the uncertainty surrounding the monitoring estimates. For example, without considering false alarms, NATO could be very aggressive in interpreting monitoring estimates as evidence of violations. However, such a policy may lead to large numbers of false accusations and a loss of confidence in the treaty. Figure 1 illustrates one possible combinations of probabilities of false alarms and missed violations. Given a particular number of inspections and other monitoring sources, any combination on the curve would be possible. By increasing NATO monitoring capabilities, e.g., increasing the number of inspections, curves closer to the origin of the graph are attained. Obviously, the closer the curve to the origin the better the NATO monituring capabilities. 
The uncertainties in the monitoring evidence can be modeled by probability distributions on the observed estimate given the actual force strength. For our analysis, we have assumed that this uncertainty can be approximated by a normal distribution. Other models of this uncertainty are possible and can be incorporated in the analysis. Our analysis indicates that using reasonable alternative classes of distribution do not affect the results in the aggregate. Figure 2 illustrates this model of the uncertainty about the monitoring evidence. The horizontal axis is the monitoring estimate of force strength. The vertical axis is the probability of an estimate. The mean $(\mathrm{m})$ of the distribution is the actual force strength. On the horizontal axis, 1 and 2 standard deviations (s) are indicated. There is a $68 \%$ probability that the estimate will fall within one standard deviation of the mean and a $95 \%$ probability that it will fall within two standard deviations.

The curve in Fig. 1, and subsequent figures, is based on relative probabilities of observing particular monitoring estimates given WTO violation and WTO compliance. If WTO is complying with the baseline declarations, then the means of all the probability distributions on the monitoring estimates will be the declared force strength. If WTO is violating the baseline declarations, then the means of some of the probability distributions on the monitoring estimates will be above the declared force strength. The relative likelihood of observing a monitoring estimate above the declared strength will then be greater if WTO is violating than if WTO is complying. The ratio of the likelihoods of the observation given violation and given compliance is a measure of the strength of the evidence in favor of violation or compliance.

\section{Analysis}

In attempting to incorporate into our analysis as many important realities of the problem as possible, we have made several assumptions as a base case. Later in this report, we will explore the sensitivities of the results to variations in these assumptions. These need to be validated before the actual required number of declared site OSIs for the baseline validation can be determined. The assumptions are:

- 2500 WTO declared sites with TLEs.

- $10 \%$ excess of any type of TLE over the total WTO declaration is the size of a violation of concern.

- The excess TLEs are spread evenly throughout the WTO bases in proportion to the declarations.

-When necessary, aerial inspections, NTM, and other monitoring provisions will be implemented so as to prevent WTO spoofing of inspections.

- The uncertainty in the estimate from an OSI of WTO forces at a single base has a 0.25 ratio of the standard deviation $(\sigma)$ to the mean $(\mu)$. This implies, for example, that if the true number of tanks was 400 , then the estimate could be modeled as in Fig. 1 where the mean is 400 and one standard deviation is 60 (i.e., there is a 68\% probability that the estimate would be between 300 and 500).

- No additional inspections are necessary to monitor sublimits, including sufficiency rule, stationed active force rule, and geographic subceilings.

- Quotas evaluated are minimum of passive and active quotas. 
- Inspection of only one base per inspection (i.e., each "inspection day" covers only one base).

The results of the base case analysis are presented in Fig. 3. Note that as few of 50 baseline OSIs can achieve a monitoring capability with less than $10 \%$ of either missing a militarily significant violation or signalling a false alarm. As illustrated, more inspections always provide improvements in monitoring capabilities, but with diminishing improvements in monitoring ability for each additional inspection.

In the following paragraphs, we explore the sensitivity of these base case results to changes in the assumptions.

\section{Sensitivity to Assumptions about Uncertainty in Inspection Estimates}

We have extended the base case analysis to examine the effects of different judgments about the uncertainty surrounding the monitoring estimates from inspections of bases. In Fig. 4, we present the results for different ratios of the standard deviation to the mean: 1.0, 0.5, 0.25 (base case) and 0.125 . Figure 4 assumes 50 inspections. With $\sigma / \mu=0.125$ the U.S. has almost perfect monitoring capabilities, but with $\sigma / \mu=1.0$, the inspections do not greatly improve upon NTM capabilities. One way to reduce uncertainties, and therefore the number of ins rections, would be to have an aerial inspection accompany each declared-site OSI.

Figure 5 is similar to Fig. 4, except Fig. 5 assumes 100 inspections of declared sites during the baseline period.

\section{Sensitivity to Assumptions about Spoofing}

In our base case, we assumed no spoofing of the inspections by WTO. This means that if the WTO were violating at a site, the mean of the probability distribution on the monitoring estimate would be the actual violating force strength. We can easily model possible WTO spoofing of inspections by reducing the mean of the probability distribution on the estimate given violation. The best WTO could hope to do, would be to reduce the mean for the estimate given violation to the declared force strength, i.e., the probability distributions on the estimate given compliance and violation would be identical. In Fig. 6, we have assumed that the WTO is able to spoof the inspections at one half of the sites visited. Compared to Fig. 3, the inspections are now less effective and more inspections are required to obtain the same level of monitoring capability. NATO may be able to prevent WTO spoofing of declared-site OSIs by coordinating the inspections with aerial overflights, suspect-site inspections, and NTM coverage.

\section{Sensitivity to Assumptions about WTO Evasion Strategy}

In evading NATO detection of an attempt to exceed the declared WTO force strengths by $10 \%$, the WTO has many alternatives for allocating its forces among bases. In our base case we made the most conservative assumption (from NATO's perspective) that the excess forces would be allocated among all the bases. Grouping the excess forces at selected bases increases the probability of NATO detection. While reducing the chance that NATO would inspect a violating site, if NATO did inspect such a site the probability that the violation would be detected increases by a relatively greater amount. This is illustrated in Fig. 7. The dashed curves in Fig. 7 assume that WTO allocates all of the $10 \%$ excess forces to the largest 900 bases. The U.S., if sites are selected randomly, then has only a $900 / 2500$ chance of selecting a violating base for any individual 
inspection. However, each of the violating bases now has $20 \%$ excess over their declared force strengths.

\section{Sensitivity to Size of Violation}

In the preceeding analysis we have taken a perspective in which we assumed a militarily significant violation and evaluated different quotas according to their verification effectiveness. We can also use our methodology to evaluate the effectiveness of a fixed quota against different sizes of WTO violations. In Fig. 8, we assume a fixed quota of 50 OSIs and, maintaining all other base case assumptions, present the effectiveness of such a quota against violations of $5 \%, 10 \%$, and $20 \%$ excess WTO forces. Note the great increase in effectiveness for a $20 \%$ violation relative to the assumed militarily significant violation of $10 \%$. Conversely, verification effectiveness is much lower for a 5\% violation. Figure 9 is similar to Fig. 8, except that it assumes a quota of 100 inspections.

\section{Extension to Annual OSIs Quotas}

The focus of this effort was on base-line validation. However, the same methodology is easily applied to annual OSI quotas during the life of the treaty. Such an extension requires consideration of a possible WTO force build-up.

For base-line validation, NATO would like to detect a militarily significant WTO evasion during the relatively short validation period. The likelihood that a significant WTO change in overall forces during this period would go undetected is probably negligible. However, during the course of the treaty a gradual build-up of forces to a militarily significant level may be a major conern. To be effective, OSIs should detect such a build-up before it reaches a miltarily significant level. To do so, OSIs will be required to detect evasions of less than the militarily significant level (e.g., less than 10\%) while the build-up is in process. A gradual build-up will not occur overnight (almost by definition), and some period of time will be required. The shorter the required period of time, the less time that OSIs have to detect the build-up, but the more likely NTM or other verification measures will detect it. Figure 10 illustrates the required number of declared-site inspections to monitor continuing WTO compliance if a militarily signficant violation is $10 \%$ excess forces and if the time required for a covert build-up is one year.

\section{Site Selection for Inspections}

In the preceding analysis, we have assumed that the U.S. would choose the base for inspection at random. This was done to be conservative. In reality, NTM, the information exchange, and the baseline declarations themselves would provide a great deal of information for targeting of OSIs. Such information, if used properly, may allow the U.S. to have a higher probability of detecting a WTO violation than allowed by purely random selection of bases. Random selection of bases for inspection has the benefit of minimizing the WTO ability to "game". the U.S. OSI selection process and further reduce the probability of detection of a violation. However, the key to random. inspections is not to actually choose randomly, but to select inspection sites in such a manner that the WTO remains uncertain as to where the next U.S. inspection selection will be. The U.S. should use all information available to select a base for inspection, always remembering to give the selection process an aura of randomness. When selection sites for OSIs based on NTM, the U.S. must be cognizant of the possibility of WTO cueing, i.e., creating signs for detection by NTM that result in the U.S. wasted an inspection. If in selecting sites for OSIs, the U.S. makes the process truly look random to the WTO, this is less of a concern. (If the U.S. site selection process looks random, the WTO would not know how to influence future site selections.) 
There are several perspectives on random sampling of sites for inspection. Some sampling policies are more vulnerable to "clever" cheating by the WTO than others. Properly choosing among the sampling options may allow the U.S. to reduce the number of OSIs required to achieve a particular detection level. The process for randomily selecting sites for OSIs can be of two types: uniform, where each site is equally likely to be selected; or proportional, where the probability of selecting a site is proportional to the force at that site. Proportional site sampling may be more effective in detecting larger violations sooner. The U.S. may also choose to sample with or without replacement. Sanıpling without replacement (i.e., without including sites that have already been visited in the population of sites for selection for future inspections) limits inspections to at most one visit per site. Otherwise, the sampling is with replacement. Depending on the U.S. sampling policy, the WTO means for evading the treaty and minimizing the probability of detection will vary. In our analysis, to be conservative, we have the assumed uniform site sampling and sampling with replacement.

\section{Summary}

This report presents a decision analysis and evaluation to assist in resolving important issues concerning the CFE declared site inspection protocol. Declared site OSIs, other treaty provided measures, and NTM are all required for effective validation of CFE baseline declarations. None would be particularly effective without the others. For this analysis, we represent the monitoring effectiveness of OSIs as possible combinations of probabilities of detecting a violation if the WTO indeed violates the baseline declarations and probabilities of falsely alarming. We assume $10 \%$ excess forces constitute a militarily significant violation. Using these results, a policy-maker can seek to maximize the probability of detecting a violation given that one occurs, while trying to minimizing the number of inspections.

In our analysis, we attempt to go beyond simple statistics and incorporate as many important aspects as possible impacting the validation of baseline force strength declarations. Many of the important aspects of the problem are subject to differing judgments. We have explored the sensitivity of the analysis to differing judgments and illustrated their effects on the results. We also included a discussion about the methodology for actually selecting a site for inspection.

The determination of the desired number of baseline declared-site OSIs requires a mix of technical and policy judgnients. This report attempts to incorporate the best technical knowledge available while leaving the policy judgments as parameters to be decided by the policy-maker. Some of the more important policy judgments are:

- Choice of the required probability of detection

- The tradeoff of increased detection against the intrusiveness of additional inspections.

- Percentage of excess forces constituting a militarily significant violation.

- Willingness to coordinate with and rely on inspections completed by NATO allies

- Need for inspections for reasons other than compliance evaluation.

Several of the technical factors in this analysis require further research and evaluation. More indepth consideration of these factors may require changes or additions to the analytical framework. Factors requiring additional work are: 
- Mechanism of inspections and how estimates of force strength should, or will, be made from observations.

- Uncertainty inherent in the monitoring estimates based on the inspections.

- Capacity and means for spoofing inspections and corresponding opportunities for coordinating or structuring verification measures to prevent spoofing.

- Necessity for coordinating declared-site inspections with aerial inspections, suspect-site inspections, and NTM.

- Number of bases covered by an inspection and the relationship between the time spent on an inspection and the uncertainty in the estimate of force strength.

- Need for additional inspections to verify sublimits .

Consideration of these factors would be natural extensions of the analysis presented above.

\section{Acknowledgments}

The authors would like to thank James Behrens and Commander Arthur Kuehne, USN, both with the Office of the Joint Chiefs of Staff, for their motivation and support of this evaluation. The authors appreciate the assistance of T.R. Koncher and P. Herman of the Lawrence Livermore National Laboratory Treaty Verification Program. 


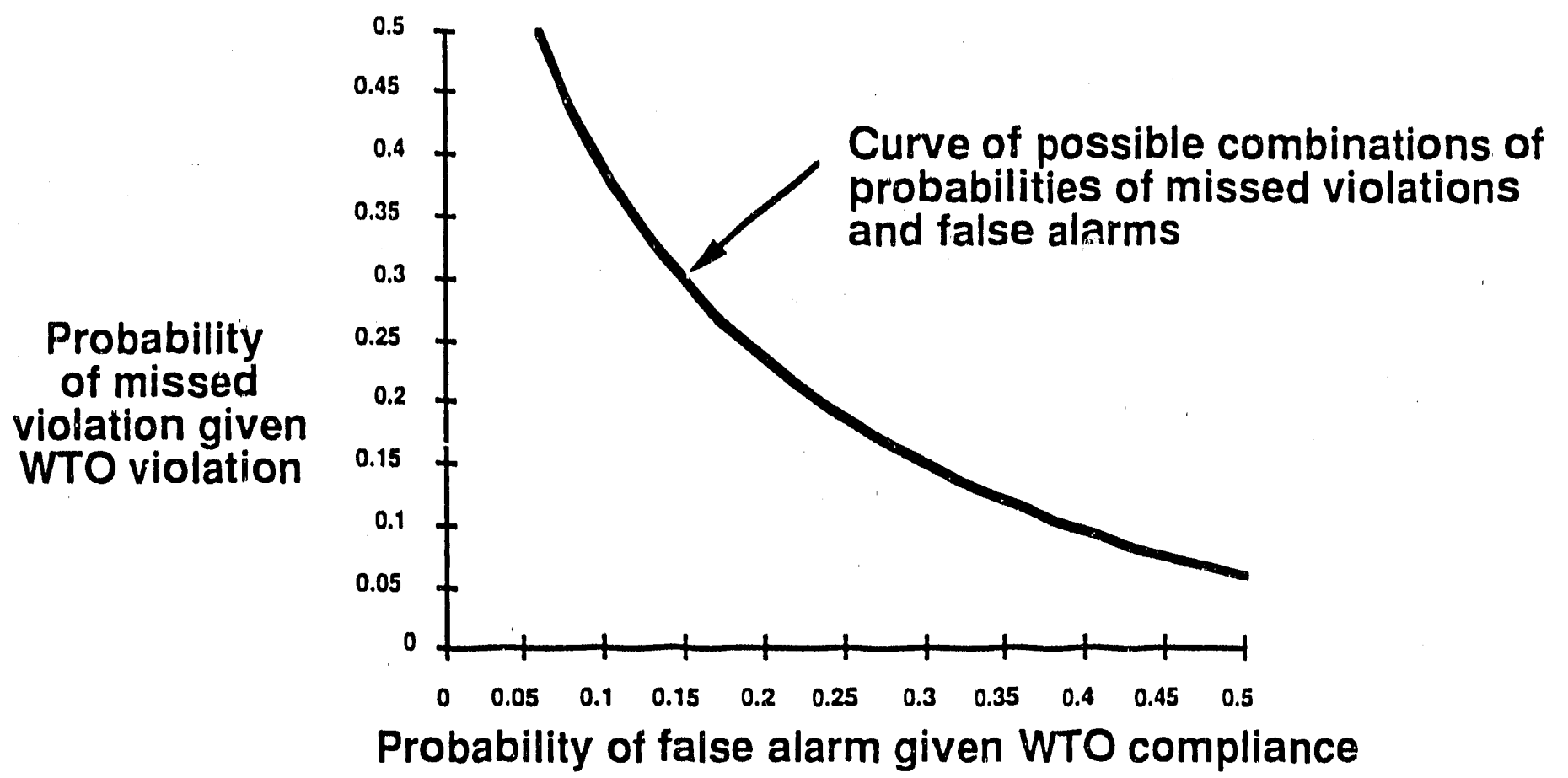

Figure 1. 
There is a $68 \%$ probability that the estimate will fall within one standard deviation $(\sigma)$ and a $95 \%$ probability that it will fall within two $(2 \sigma)$

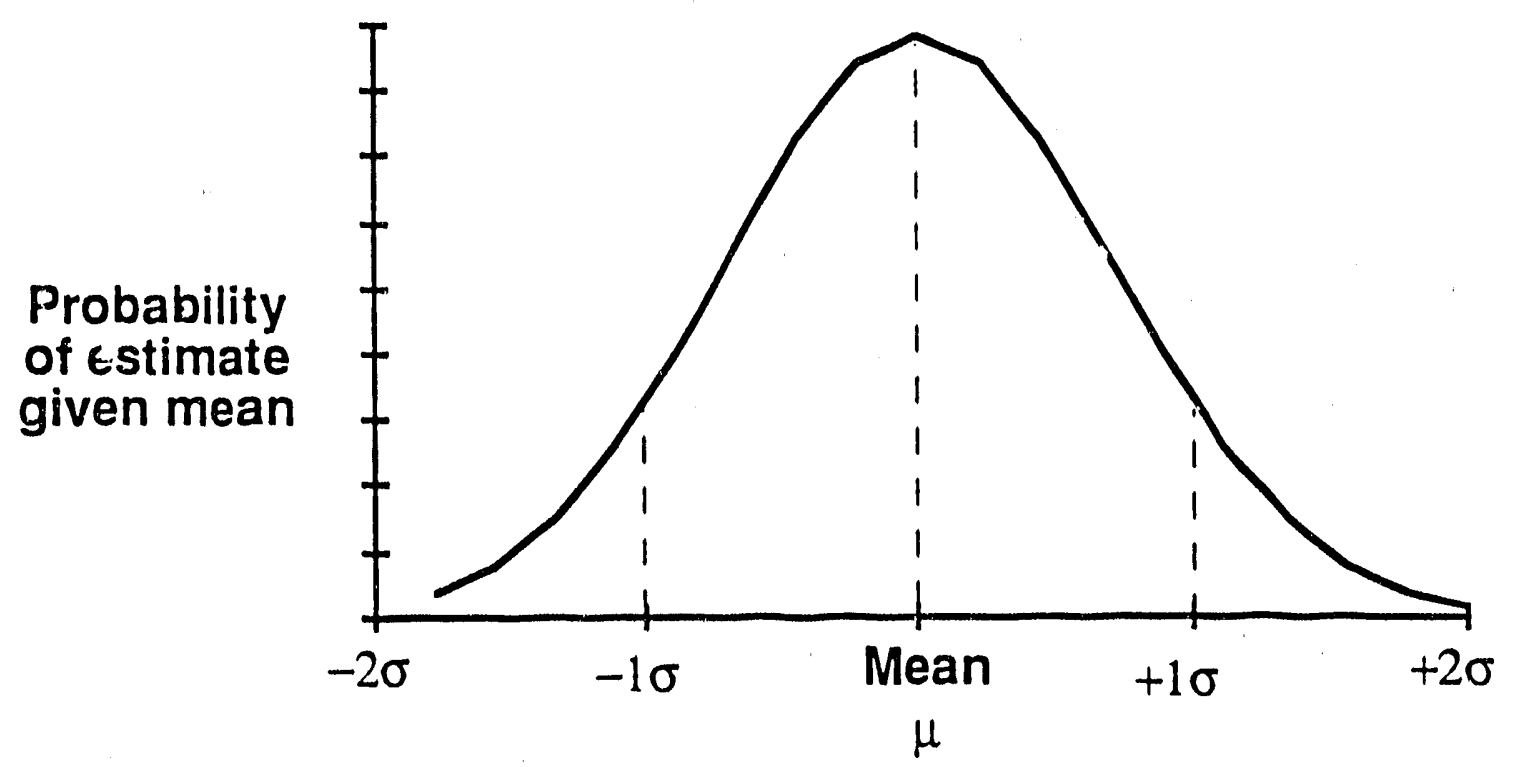

Estimate of force strength

Figure 2. 


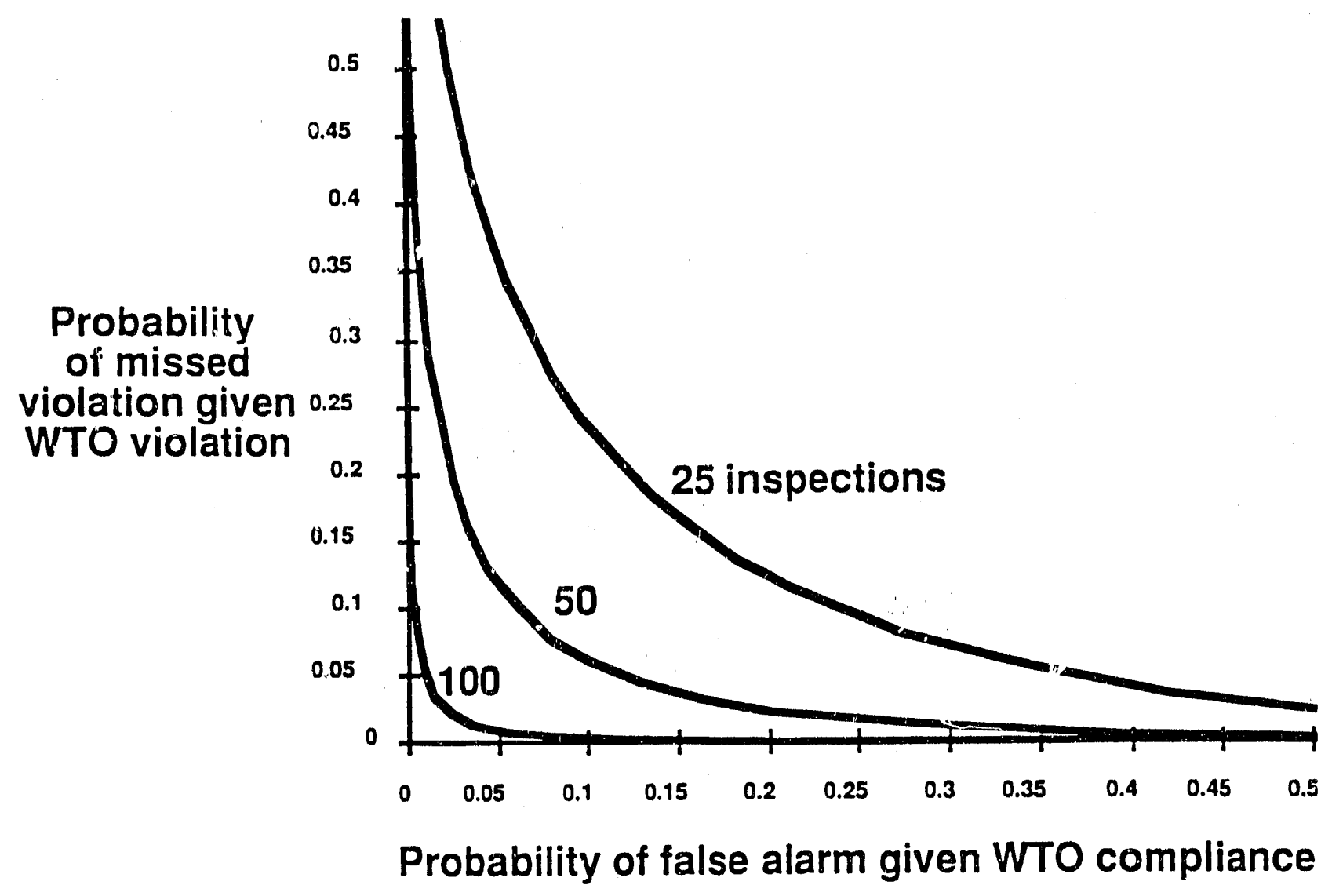

Figure 3 . 


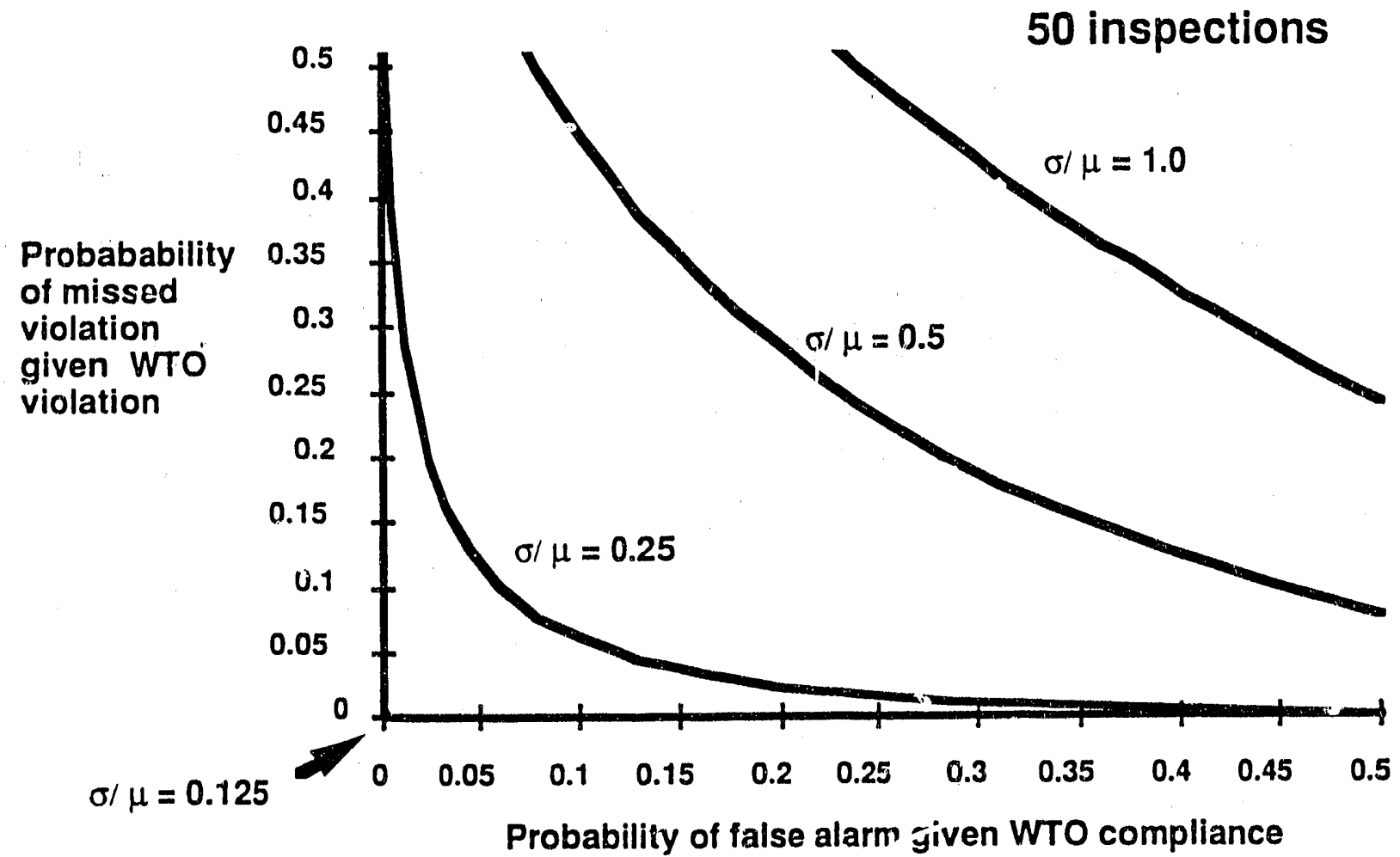

Tigure 4. 


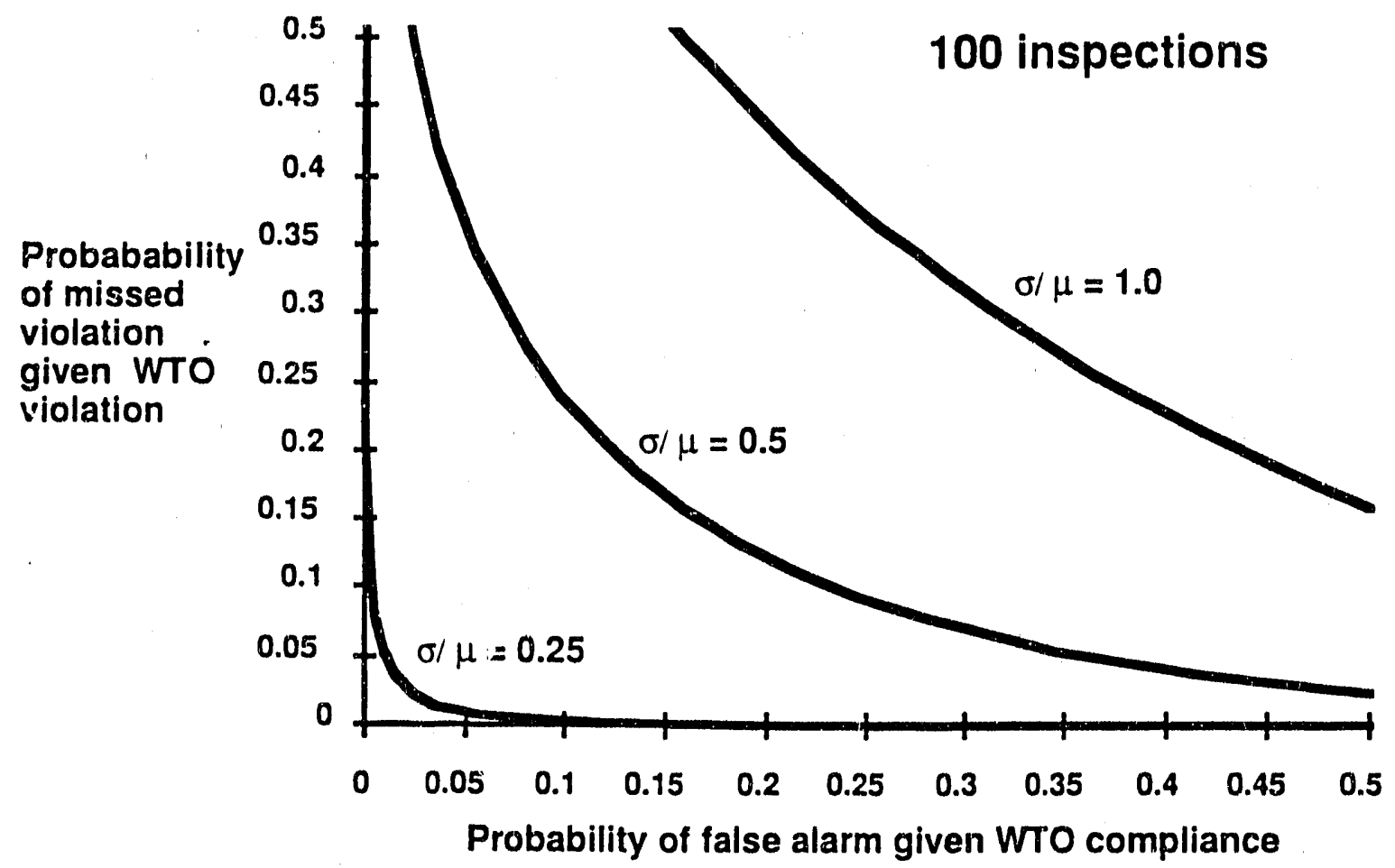

Figure 5. 


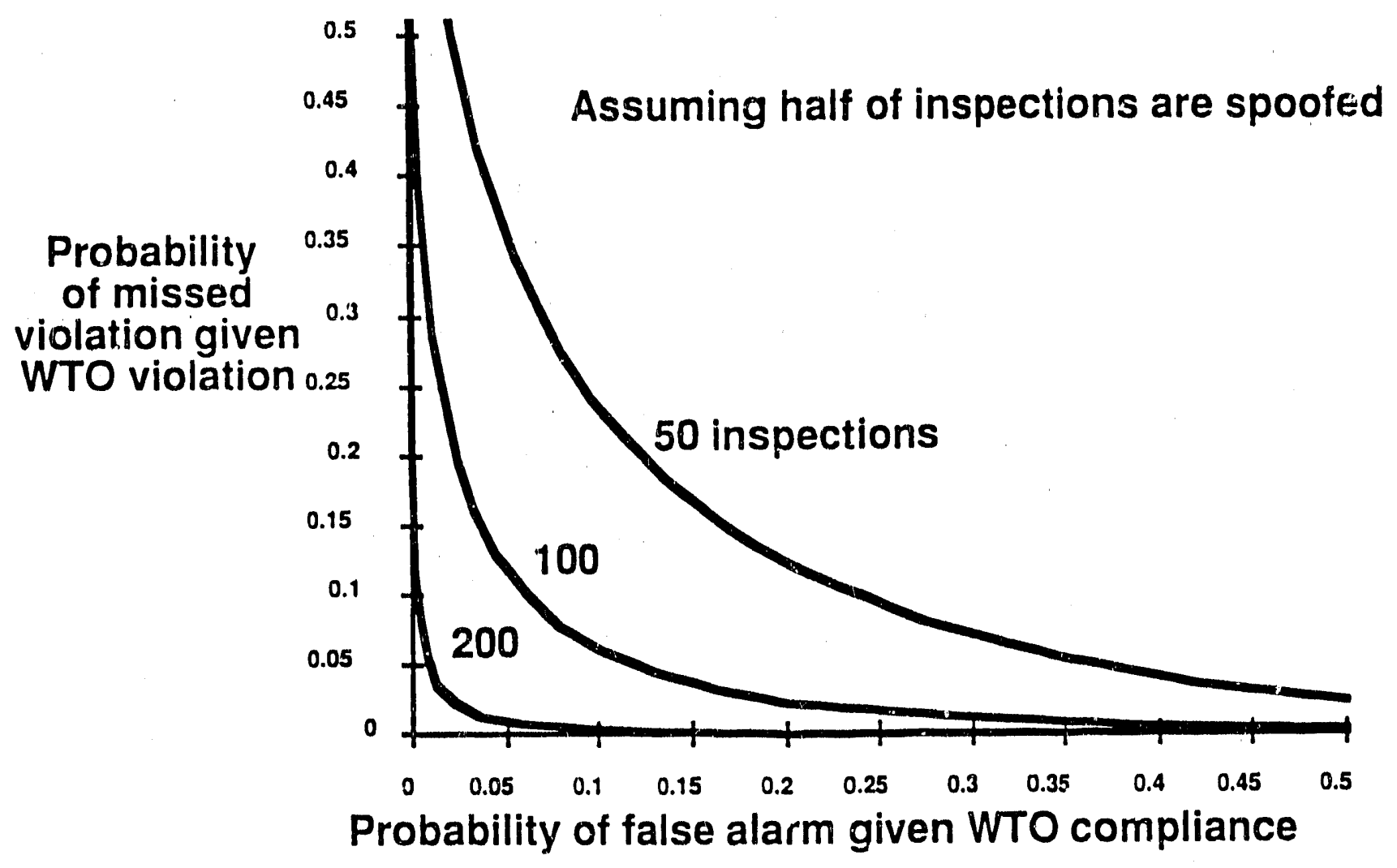

Figure 6. 


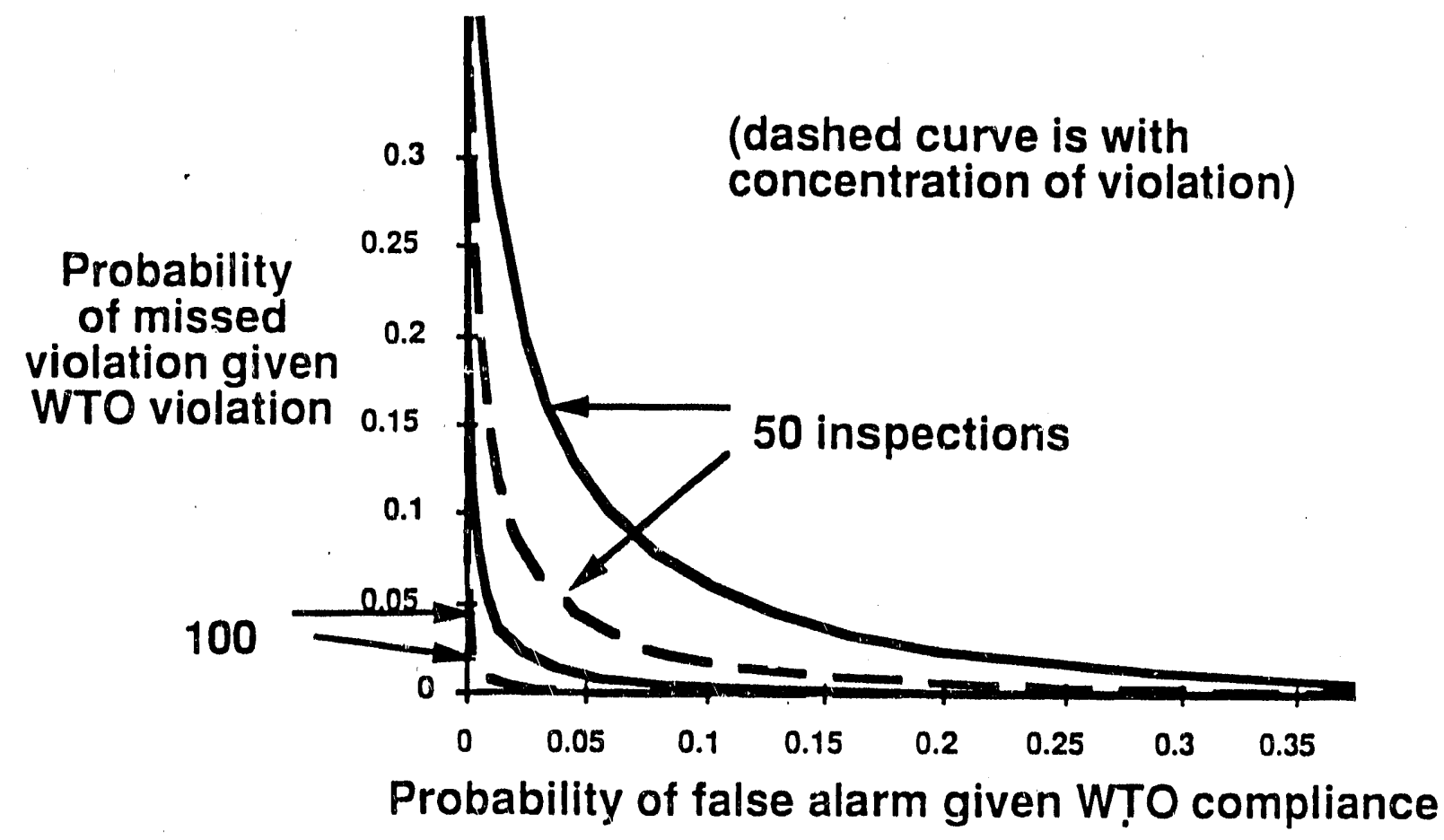

Figure 7. 


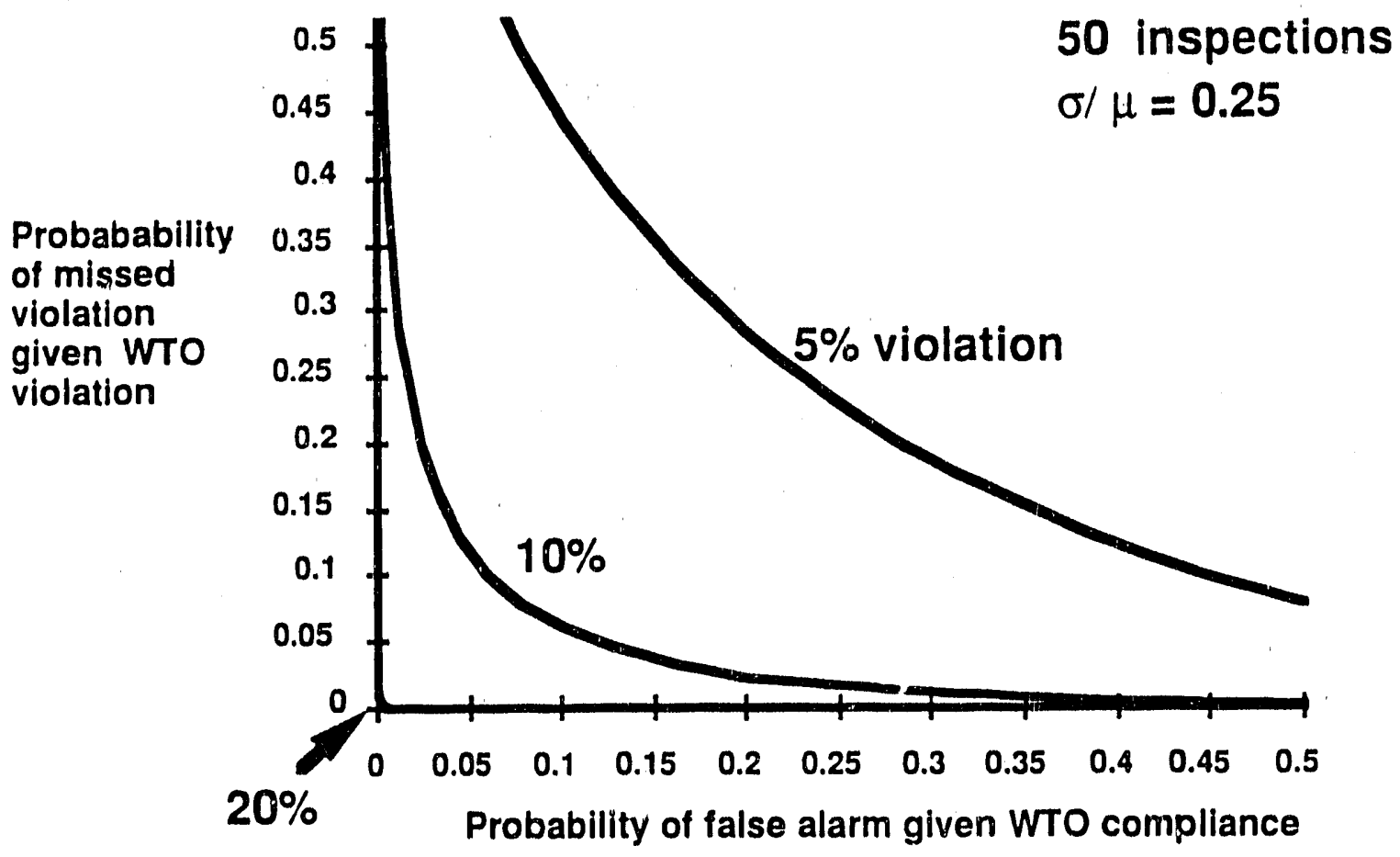

Figure 8. 


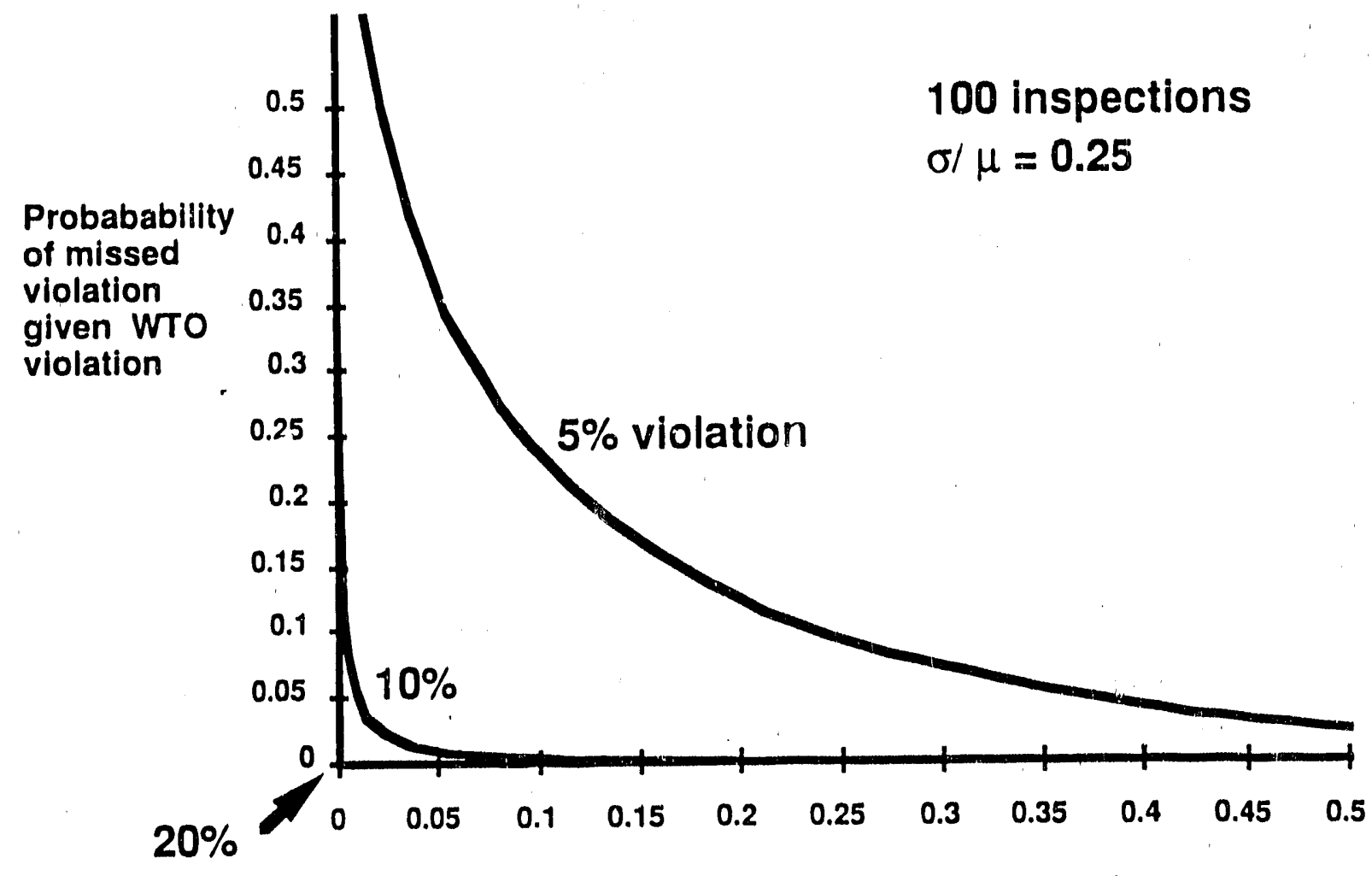

Probability of false alarm given WTO compliance

Figure 9. 


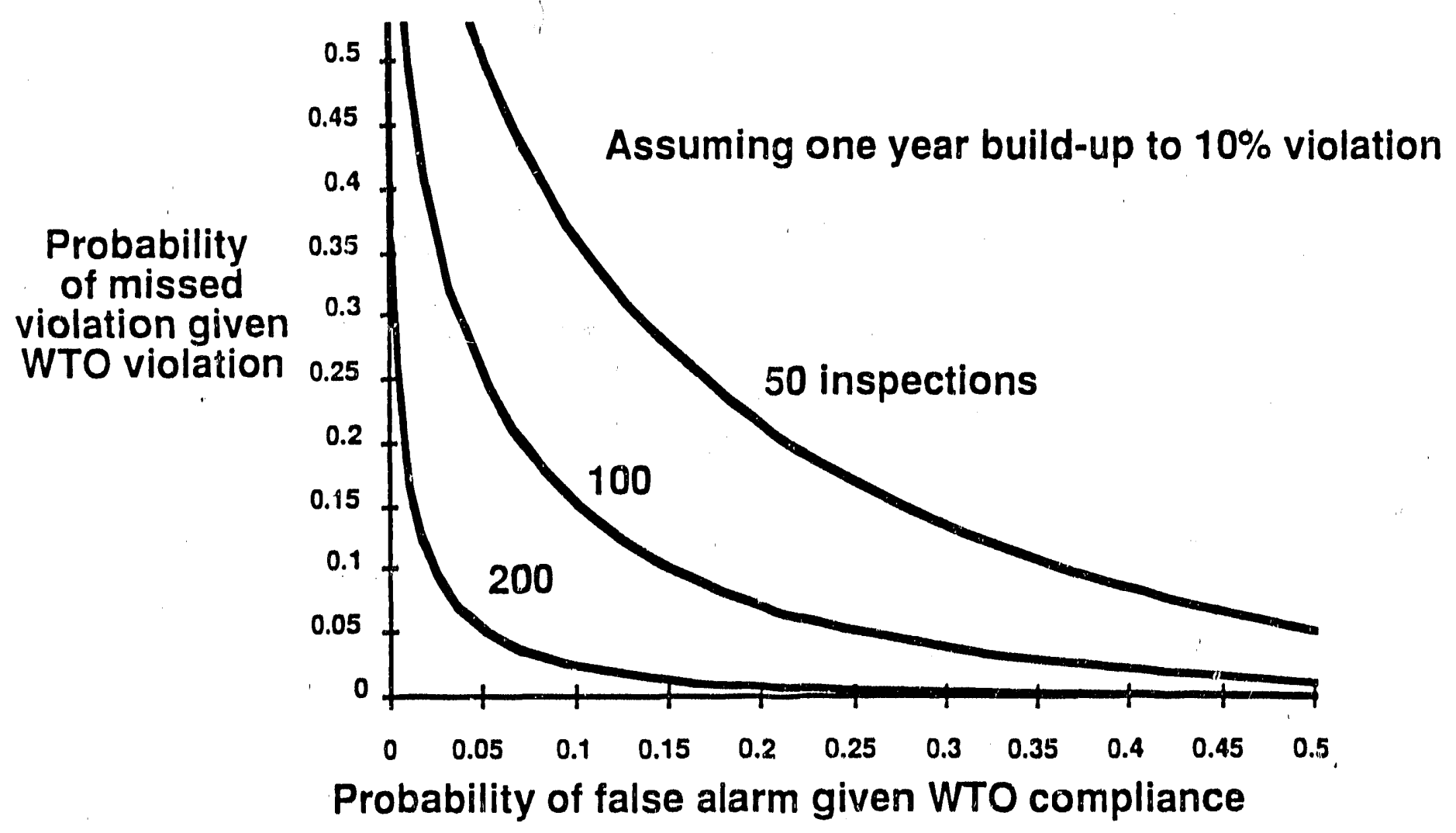

Figure 10. 

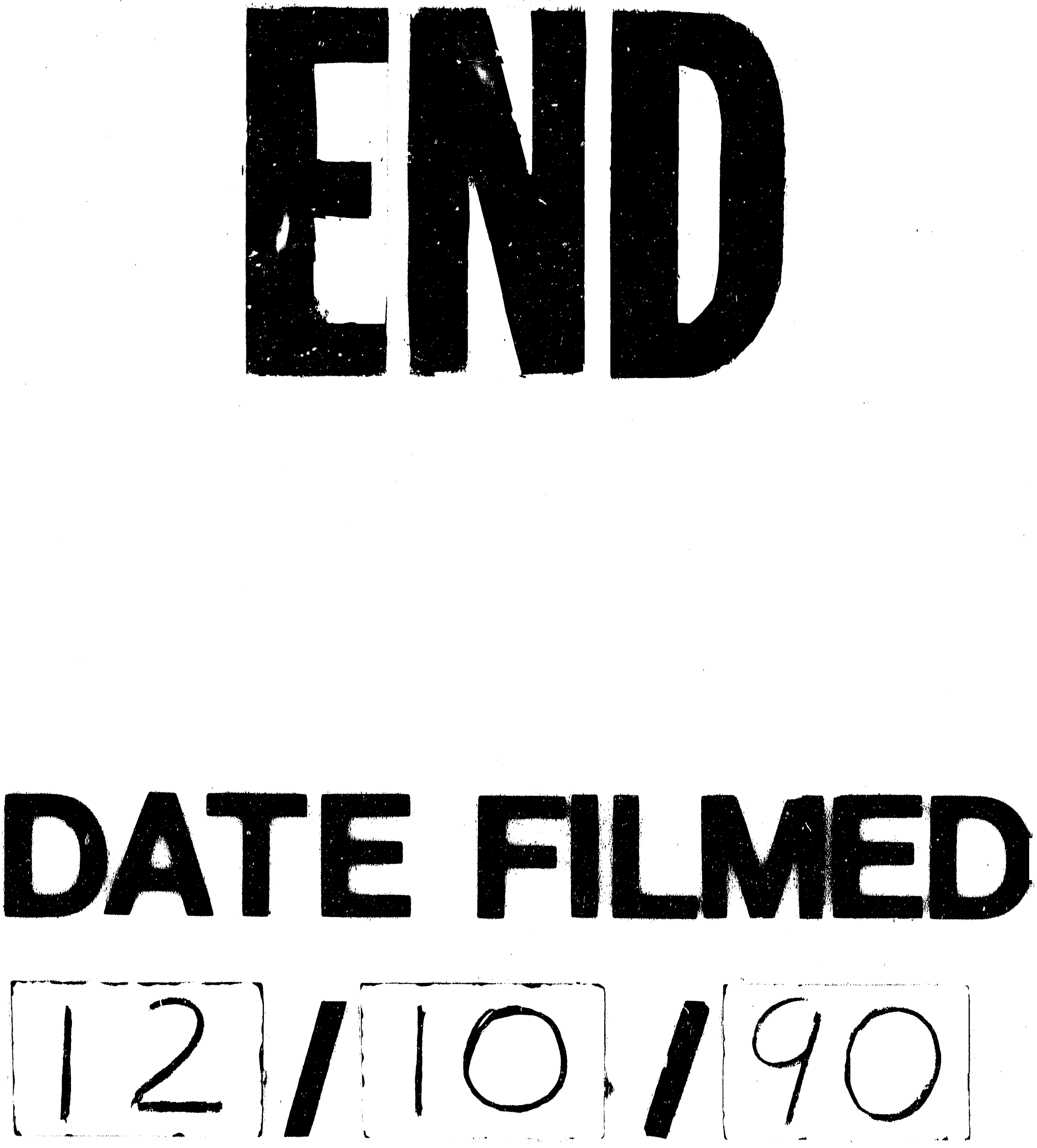
